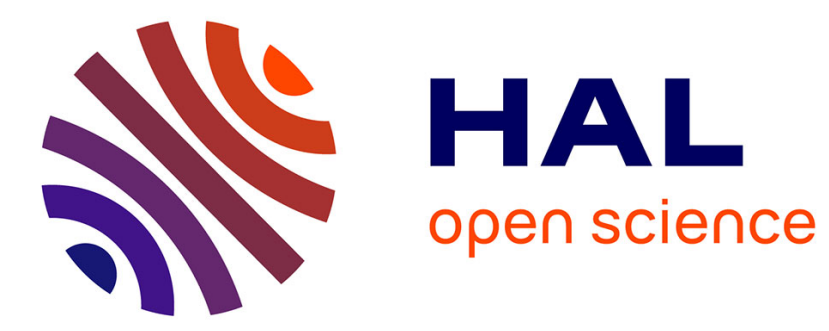

\title{
La typologie des stations forestières. Recommandations méthodologiques
}

\author{
Alain Brêthes
}

\section{To cite this version:}

Alain Brêthes. La typologie des stations forestières. Recommandations méthodologiques. Revue forestière française, 1989, 41 (1), pp.7-27. 10.4267/2042/25949 . hal-03424651

\section{HAL Id: hal-03424651 \\ https://hal.science/hal-03424651}

Submitted on 10 Nov 2021

HAL is a multi-disciplinary open access archive for the deposit and dissemination of scientific research documents, whether they are published or not. The documents may come from teaching and research institutions in France or abroad, or from public or private research centers.
L'archive ouverte pluridisciplinaire HAL, est destinée au dépôt et à la diffusion de documents scientifiques de niveau recherche, publiés ou non, émanant des établissements d'enseignement et de recherche français ou étrangers, des laboratoires publics ou privés. 


\title{
LA TYPOLOGIE DES STATIONS FORESTIÈRES Recommandations méthodologiques
}

\author{
A. BRÊTHES
}

La réalisation d'une étude des stations forestières, sur un massif ou une région naturelle, doit aboutir à la fois à l'établissement d'une référence scientifique et d'un outil de travail pour le forestier.

L'accroissement du nombre de ces études et la grande diversité des documents publiés ont rapidement rendu nécessaire de préciser les besoins des forestiers, de réfléchir aux moyens de répondre à ces demandes et d'élaborer dans le domaine des études de stations forestières un langage commun compréhensible par tous.

S'est ainsi constitué, il y a quelques années, un groupe de travail rassemblant toutes les parties prenantes dans ces études - des scientifiques aux forestiers - (Dumé, 1988). Au sein de ce groupe, une commission a été particulièrement chargée de préciser les buts relatifs à la typologie des stations forestières et de définir les moyens d'y parvenir. II ne s'agissait en aucun cas d'édifier une norme détaillée devant être utilisée par tous, mais d'élaborer certains principes méthodologiques, des recommandations, voire des directives, qui assureraient la bonne fin de ces études.

Le présent article reprend l'essentiel du fruit des travaux de cette commission sous forme de rappels ou de précisions de certains éléments indispensables (voire obligatoires) pour mener à bien de telles études dans des peuplements forestiers bien constitués et proches de l'équilibre naturel. Le cas des formations très artificialisées ou dégradées fait encore l'objet de discussions et de tests méthodologiques.

\section{RAPPELS DE QUELQUES NOTIONS FONDAMENTALES}

Nous n'entrerons pas dans le détail des définitions de base qui ont été rappelées par ailleurs (Becker, 1988 ; Becker et Le Goff, 1988), mais il est nécessaire de préciser certaines notions dont la connaissance est indispensable pour comprendre les options prises en matière de méthodologie. 


\section{A. BRÊTHES}

\section{La typologie des stations forestières}

On entend par typologie la reconnaissance et la description de tous les types de station d'un massif forestier ou d'une règion forestière.

Cette typologie doit fournir au forestier gestionnaire :

- d'une part, les éléments, si possible simples, de caractérisation des types de station de son massif (caractérisation ponctuelle ou à but cartographique) ;

- d'autre part, des informations suffisantes pour l'évaluation des potentialités de production (réserve en eau, niveau de nutrition minérale, contraintes majeures ou secondaires, etc...) de chacun de ces types de station. Une étude des stations qui ne comporterait pas les informations écologiques et biologiques permettant le choix des essences à favoriser et la compréhension de leur comportement serait inutilisable en gestion.

Becker (1985) soulignait que: "les études de station sont par nature même très pluridisciplinaires, et s'appuient largement sur l'écologie générale, l'écologie végétale, la botanique, la phytosociologie, la pédologie, la géomorphologie, la géologie, la climatologie..." . Ainsi ce serait une erreur de concevoir comme suffisamment précise une typologie qui se baserait exclusivement sur une étude floristique sans tenir compte du facteur sol ou qui, inversement, ne comporterait qu'une description sommaire des types de sol.

- Pourquoi une étude à l'échelle régionale?

Chaque massif forestier n'offre qu'une vision partielle de l'ensemble des stations forestières d'une région. Réaliser une étude pour chaque massif pris isolément conduit à répéter plusieurs fois la même démarche et, parfois, de manière superficielle, avec des moyens limités. De plus la comparaison des résultats d'un massif à l'autre sera toujours délicate.

L'étude régionale permet, en raison de l'importance des surfaces concernées, de recourir à des spécialistes de ce type d'étude et de disposer d'un budget en conséquence. Mais surtout, les résultats d'une telle étude constituent un langage commun à tous les forestiers, publics ou privés, de la région, leur permettant de mettre en commun leurs expériences et d'en tirer le meilleur profit.

- Le catalogue des stationis forestières

C'est le "produit fini " de l'étude de typologie régionale. La rédaction et la présentation d'un tel document doivent répondre à deux finalités qui peuvent, à première vue, paraître contradictoires :

1) Si le catalogue des stations ne doit pas tomber dans le travers d'une trop grande complexité scientifique, il doit toutefois ne pas craindre d'être complet, même si tous les résultats acquis peuvent ne pas être utilisès dans l'immèdiat.

La typologie doit refléter la diversité écologique de la région. Elle doit être aussi exhaustive que possible. Ainsi il est illusoire de vouloir fixer à l'avance le nombre des types de station à retenir. II est hors de question de passer sous silence certains types reconnus sous prétexte qu'ils ne représentent que des surfaces réduites ou n'ont qu'un intérêt sylvicole actuellement limité.

Ce catalogue constitue un document de référence auquel autant forestiers praticiens que tous ceux qui s'intéressent à la forêt (scientifiques, enseignants, etc...) peuvent se reporter.

On devra donc éviter de prèsenter des documents trop superficiels, trop synthétiques ou trop schématiques; il est toujours plus facile (et le catalogue doit donner des éléments pour cela) de regrouper divers types de station en fonction des objectifs recherchés (station convenant au Chêne ou au Frêne par exemple) que de chercher à faire des divisions a posteriori. 
2) Mais le catalogue est aussi un outil de travail qui doit pouvoir être mis en application, après une phase d'initiation, par des personnels de niveau technicien.

Ce document devra donc être présenté de manière claire et précise. Son auteur devra faire un effort de synthèse pour ne retenir que le minimum de critères efficaces pour le diagnostic stationnel et pour présenter ceux-ci de la façon la plus opérationnelle possible: tableaux synthétiques, schémas et surtout clés d'identification. Nous y reviendrons.

Le contenu minimal indispensable et le mode de présentation d'un catalogue des stations ont été définis de manière très précise par la commission "méthodologie". Ces propositions ont été rassemblées dans une brochure rédigée par J. Timbal : "Recommandations pour la présentation des catalogues des stations forestières " (1984). II est indispensable de s'y référer pour toute rédaction de ce type de document.

\section{- La notion de région naturelle}

En raison des diversités climatiques et géologiques de notre pays, il n'est pas pensable de vouloir réaliser des typologies stationnelles, mème simplifiées, pour de vastes territoires et encore moins à l'échelle de la France. Un découpage géographique régional s'impose autant pour des raisons très matérielles de mise en cuvre de l'étude, que, surtout, pour des raisons scientifiques parmi lesquelles on retiendra celles qui intéressent la végétation: chorologie, existence d'écotypes, compensations de facteurs, etc (Becker, Le Goff, 1988).

Les résultats de la typologie (détermination des types de station, liaison entre types de station...) et des études ultérieures (liaisons station-productivité) doivent pouvoir s'appliquer sans réserve en tout point de la région retenue.

Le cadre idéal pour une telle étude est celui de la " petite région naturelle " (Becker, 1986), par exemple: Perche, Argonne, Plateau lorrain, Châtaigneraie limousine, etc.

Ce découpage régional pourra s'appuyer sur ceux déjà existants: en particulier régions forestières de l'Inventaire forestier national, mais aussi régions naturelles des géographes, carte des régions écologiques de Dupias et Rey, etc.

Une telle région naturelle comprend à la fois des forêts privées et des forêts "soumises ". II est évident que la typologie des stations concerne à la fois l'une et l'autre catégories de forêts. La réalité du terrain et la nécessité scientifique font qu'en toute logique, il est indispensable que l'échantillonnage des points de relevés se fasse sur l'ensemble des massifs. Cette façon de procéder peut d'ailleurs être enrichissante en décelant de fines variations (par exemple de la composition floristique) qui relèvent de modes de gestion différents.

La région naturelle est également indépendante des structures administratives; on peut être amené à réaliser une étude dans une région à cheval sur plusieurs départements ou régions administratives. Certes une telle situation ne facilitera pas les liaisons entre le réalisateur et les divers services forestiers concernés, mais il serait regrettable d'arrêter une région naturelle à une limite administrative imposée par le demandeur.

\section{Station, type de station et sylvofaciès}

La station est une unité concrète de terrain correspondant à des conditions écologiques locales sensiblement homogènes dépendant de la nature des formations géologiques superficielles, du climat et de la topographie. Ces conditions s'expriment à travers le sol et la végétation. 


\section{A. BRÊTHES}

Lors des études de typologie, la station sera donc caractérisée par un mésoclimat, un groupement végétal et un type de sol qui lui sont propres.

La station n'a pas une superficie déterminée. Cette dernière dépend du contexte local (versant, plateau, etc...), du degré d'hétérogénéité que l'on peut accepter pour certaines de ses caractéristiques et donc de la précision que l'on recherche. On peut ainsi reconnaitre des microstations: la base d'une cépée de Charme, le bord d'un fossé de drainage, le talus d'un chemin, etc... Si ces microstations peuvent être d'un grand intérêt scientifique, elles sont inutilisables dans la gestion forestière. II n'existe cependant pas d'aire minimale préconisée. Lors de l'établissement de la typologie, pour être certain de la précision du document final, on peut donc être amené à échantillonner des unités n'ayant que quelques ares de surface.

Le type de station, en revanche, n'existe pas en tant que tel sur le terrain. C'est un concept, c'est-à-dire une unité de classification théorique qui regroupe l'ensemble des stations analogues par la position topographique et géomorphologique, la nature du sol, la composition floristique et la dynamique de la végétation, etc...

Un type de station est donc, par définition, indépendant de la nature du peuplement actuel ; certes il correspond à un peuplement climacique potentiel mais rarement observable dans nos forêts fortement anthropisées. Ainsi un même type de station peut exister sous Chênes et sous Pin sylvestre, voire sous une friche ou une prairie (Rameau, 1986).

Si les caractéristiques fondamentales du type de station (situation topographique, nature des formations superficielles ...) sont stables dans le temps, d'autres peuvent évoluer et donc se modifier, notamment en fonction des traitements sylvicoles: composition et structure du peuplement, végétation naturelle (appauvrissement, banalisation), et même, type d'humus (cas des enrésinements). On peut considérer que tant que ces modifications n'ont pas d'incidence sur la potentialité forestière et qu'elles sont réversibles, on a toujours affaire au même type de station. On nomme alors sylvofaciès les différentes "physionomies prises par un même type de station lorsque la sylviculture éloigne son peuplement du climax " (Delpech et al., 1985).

La notion de type de station est également indépendante de la notion d'échelle; le type de station est défini en tant qu'unité écologique homogène quelle que soit son extension spatiale. Toutefois, les contraintes de gestion forestière exigent de ne pas faire de pointillisme; on doit rechercher à définir des types ou des groupes de stations ayant une certaine représentativité spatiale, tout en restant suffisamment homogènes.

\section{DÉMARCHE MÉTHOdOLOGIQUe PROPOSÉE}

II n'est pas dans notre propos, comme nous l'avons indiqué en introduction, de décrire une méthode dans ses moindres détails et encore moins de vouloir l'imposer.

Cependant, compte tenu des résultats des études régionales actuellement disponibles (Dumé, 1987), et des six années de réflexion de la commission "méthodologie ", il apparait possible et même nécessaire de proposer une démarche générale cohérente, même si certains aspects méritent encore d'être précisés. Pour une large part, cette démarche découle d'ailleurs implicitement du contenu exigé pour les catalogues de stations.

L'assurance de bien atteindre le but assigné à ce type d'étude nécessite que tout au long de celle-ci, depuis la préparation de l'échantillonnage jusqu'au dépouillement des données et à l'établissement de la typologie, une certaine rigueur scientifique soit respectée. 
Mais rigueur ne signifie pas uniformité. Étant donné que chaque région présente des caractères particuliers quant à son climat, ses roches mères, sa flore, ses corrélations sols-végétation, etc.... il serait utopique de vouloir imposer une méthode d'étude stricte. Chaque réalisateur a, au contraire, la responsabilité de mettre en avant les facteurs qui lui paraissent les plus discriminants pour caractériser les stations forestières de sa région, en partant du principe que la typologie en elle-même repose sur la connaissance de l'ensemble des facteurs du milieu (en particulier climat, topographie, végétation, sol).

Rappelons que, pour qu'elle soit applicable à tous les niveaux de la gestion forestière, toute étude typologique régionale doit avoir comme résultats essentiels:

- la définition des divers types de station de la région concernée;

- la description exacte, précise et complète de ces divers types (situation topographique, sol, groupement végétal et dynamisme de la végétation, peuplements existants, etc...) et de leurs sylvofaciès;

- la précision, pour chaque type, des facteurs ayant une influence sur la croissance des arbres (fertilité, réserve en eau, possibilité ou contrainte pour l'enracinement, mésoclimat, etc...) et des améliorations possibles (travail du sol, fertilisation, drainage...);

- la mise en évidence des caractères simples de reconnaissance (floristiques, pédologiques ou autres) permettant la réalisation rapide du diagnostic stationnel et de la cartographie.

Ces diverses informations permettront la rédaction d'une fiche d'identité pour chacun des types de station reconnus.

La mise en œuvre d'une telle typologie nécessite de travailler par étape dans un ordre chronologique précis. Nous présentons ici cet ordre et succinctement, le contenu de chacune de ces étapes, c'est-à-dire les grands traits de la démarche et les éléments indispensables pour mener à bien l'entreprise.

Une analyse plus détaillée et plus complète des démarches possibles sera présentée dans un guide méthodologique actuellement en cours de rédaction par la commission.

\section{La préétude}

En dehors de ses aspects administratifs (mise en place de l'étude, relations entre organismes forestiers, plan de financement, etc...) (Dumé, 1984), la préétude a pour but de faire le point d'une part sur la connaissance acquise du milieu régional (climat, géologie, pédologie, flore...), d'autre part sur les torêts (histoire, modes de gestion actuelle, propriétés, problèmes particuliers, etc...).

Cette préétude sera donc au début essentiellement bibliographique: il s'agit de rechercher et d'exploiter tous les documents disponibles relatifs aux conditions naturelles de la région et à ses forêts.

Par la suite, il pourra apparaître nécessaire de compléter ces informations par diverses observations de terrain: précision de la nature des formations superficielles par exemple, ou ébauche de corrélations entre facteurs ou descripteurs du milieu et végétation.

Elle conduira à la rédaction d'une monographie régionale.

A partir de ces informations, on doit être en mesure :

- de préciser les limites exactes de la région ou de corriger celles proposées par le demandeur de l'étude. Rappelons à ce sujet que la région ne doit être ni trop petite (pour que s'applique l'idée du catalogue en tant que langage commun à un ensemble important d'utilisateurs), ni trop vaste (pour éviter la dérive de la signification de certains descripteurs): 


\section{A. BRĖTHES}

- de faire apparaître les divers critères qu'il faudra prendre en compte lors de l'étude de typologie ;

- de préparer le plan d'échantillonnage (mode d'échantillonnage, stratification, disposition, etc...) et la fiche de relevé ;

- d'établir le devis et le programme de travail pour la réalisation du catalogue.

Ainsi la préétude n'est en principe pas un aboutissement mais la première étape indispensable de l'établissement du catalogue.

\section{Le travail de terrain}

La phase d'acquisition des données nécessaires à l'élaboration de la typologie est certainement la plus longue et la plus coûteuse ; de sa bonne réalisation dépendront totalement la qualité et la précision des résultats.

Le mode d'échantillonnage (disposition des points d'observation dans l'espace) et le contenu du relevé phyto-écologique devront, comme nous venons de l'indiquer, avoir été définis à l'issue de la préétude.

S'ils peuvent être adaptés à chaque configuration régionale, ils doivent néanmoins respecter un minimum de règles garantissant leur fiabilité.

\section{- Mode d'échantillonnage}

Quel qu'il soit, le mode d'échantillonnage adopté doit permettre d'approcher l'ensemble de la variabilité écologique de la région.

L'échantillonnage concentré sur une ou plusieurs "forêts pilotes " révélées, par la préétude, comme largement représentatives de cette variabilité régionale offre divers avantages: réduction des temps de déplacement, réalisation possible sur une durée limitée (stage d'étudiant par exemple), meilleure compréhension des liaisons spatiales entre types de station, possibilité de dresser d'emblée une carte des stations forestières. Mais l'extension de ces résultats à l'ensemble de la région, les vérifications nécessaires, les compléments obligatoires nécessitent une deuxième phase de terrain qui reste encore importante et longue. De plus, peu de régions naturelles se prêtent à cet emploi strict du massif test en raison de la dispersion des surfaces forestières.

Il semble donc préférable de choisir un mode de répartition couvrant l'ensemble de la région.

Parmi l'ensemble des autres modes d'échantillonnage possibles, l'échantillonnage par transects apparaît comme le plus performant. II permet à la fois de comprendre le déterminisme du passage d'une station à l'autre et de stratifier l'échantillonnage en tirant parti des connaissances acquises au cours de la préétude sur le sens de variation des caractères du milieu.

Comment choisir ces transects?

Les transects doivent être implantés de façon à rendre compte au mieux de la variation reconnue. Dans la plupart des cas, ils devront tenir compte des gradients climatiques (s'il en existe), des étages géologiques, des types de matériaux, de la topographie, de la nature des peuplements ou de tout autre facteur qui se serait révélé important au niveau régional.

II n'y a pas de règle fixe à imposer pour la disposition de ces transects dans la région. D'une manière générale, les transects seront des portions de droite, plus ou moins longues, judicieusement positionnées dans chacun des massifs. Ils ne se situent donc pas obligatoirement le long d'une même ligne traversant toute la région; toutefois, lorsqu'il existe un gradient climatique 
régional, il semble intéressant de tracer un axe ou une bande parcourant ce gradient et d'asseoir sur celui-ci les divers transects en tenant compte alors des autres facteurs de variation.

Comment choisir les relevés le long de ces transects?

Une trop grande rigidité dans le choix d'un mode de répartition des relevés le long de chaque transect peut conduire à un sous-échantillonnage de certaines stations (vallons par exemple) ou à un sur-échantillonnage d'autres.

Plutôt qu'une répartition à intervalle fixe ou stratifié prévue à l'avance, il peut sembler préférable de choisir les points d'observation "à vue": au cours du cheminement, on effectue un relevé chaque fois que quelque chose change significativement dans le paysage (composition du groupement végétal, topographie, nature et structure du peuplement, etc...).

Mais, pour éviter le risque de laisser échapper certaines variations, comme la nature du substrat, qui ne se reflèteraient pas dans le paysage, nous préférons combiner la méthode à intervalle fixe et la méthode à vue: on réalise un relevé chaque fois que l'on constate une modification sensible du paysage (sans fixer de pas minimal; certains points pourront être distants de 30 ou 40 mètres) mais si aucune variation n'est décelée, on s'arrête quand même au bout d'une distance fixée d'avance (pas maximum entre deux relevés : 200 mètres par exemple).

\section{Le relevé phyto-écologique}

II convient de rappeler la nécessité de bien séparer l'élaboration de la typologie et l'utilisation de l'outil de travail que doit permettre cette typologie. Ainsi, si l'étude typologique doit faire apparaitre un nombre limité de critères simples permettant de caractériser avec exactitude les divers types de station, leur nombre et leur nature ne peuvent être fixés d'avance.

Lors de la phase d'élaboration, il est primordial d'éviter toute impasse sur l'une ou l'autre des trois composantes essentielles de la station: la topographie, la végétation et le sol.

Chaque relevé devra donc comporter au minimum et de manière obligatoire les rubriques suivantes :

- Une description de la topographie : situation, environnement, pente, exposition, altitude, avec si possible, un schéma permettant de visualiser ces divers facteurs et, par la suite, de relier les stations les unes aux autres.

- Un relevé exhaustif de la végétation, par strate, selon la méthode phytosociologique classique avec, pour chaque espèce, notation du coefficient d'abondance-dominance ou de recouvrement. Ce relevè doit comprendre, dans la mesure du possible, les bryophytes humicoles et terricoles. Pour les stations riches en espèces vernales, un deuxième passage au printemps peut s'avérer nécessaire.

- Une description précise du sol sur une petite fosse pédologique de 30 à $40 \mathrm{~cm}$ de profondeur complétée par sondage à la tarière. Les principaux caractères qui doivent être obligatoirement notés sont: le type d'humus, l'épaisseur, la texture, la structure et la pierrosité de chaque horizon, la présence éventuelle de traces d'hydromorphie, de calcaire, de phénomènes de podzolisation, l'enracinement, etc...

Sur cette placette, on effectuera également des observations concernant le peuplement (par exemple: nature, structure, recouvrement, vigueur, ètat sanitaire...). Ces intormations seront utiles pour permettre, lors du dépouillement, les comparaisons entre relevés, la compréhension de certaines variations (densité du tapis végétal, présence ou absence de certaines espèces végétales, type d'humus...), et la définition des sylvofaciès. 


\section{A. BRÊTHES}

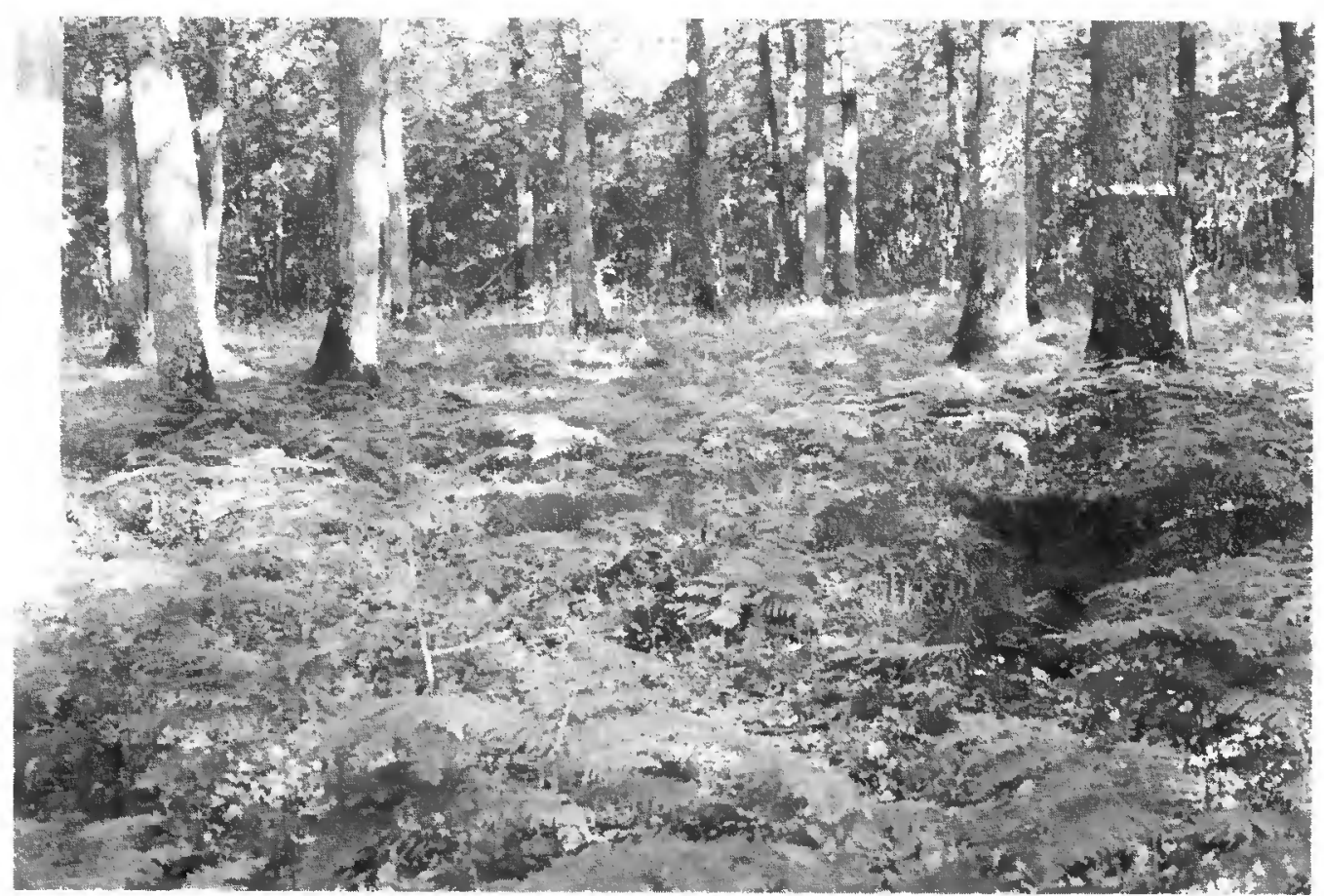

Placette de relevé avec fosse pédologique (ci-dessus). Chaque relevé comportera: « un relevé exhaustif de la végétation * (ci-dessous, à gauche) et * une description détaillée du sol » (ci-dessous, à droite).

Photos A. BRÉTHES

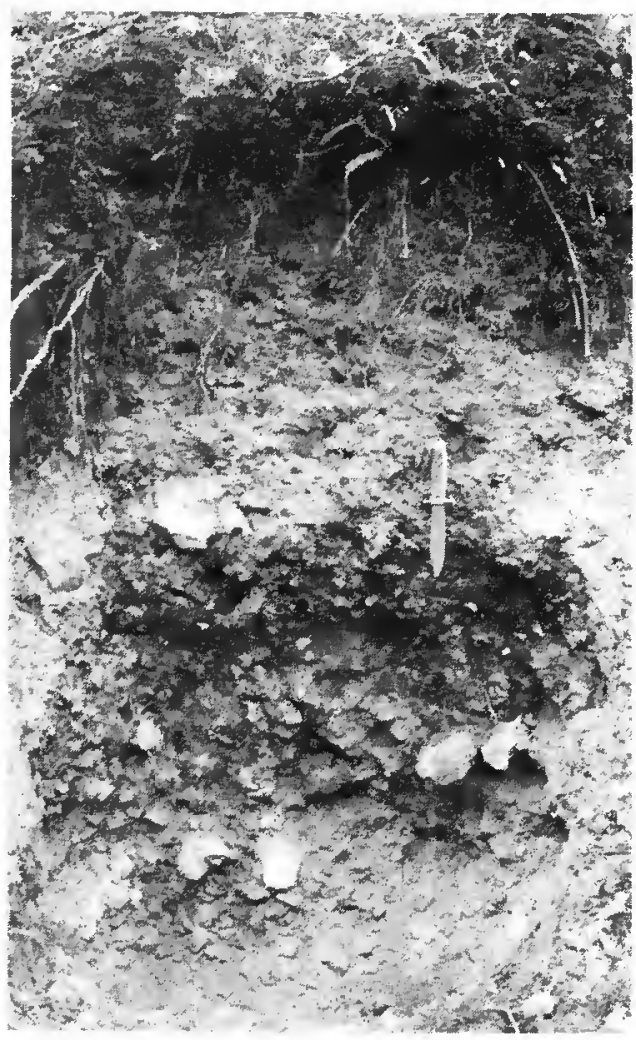


II n'est pas obligatoire, à ce stade de l'étude, d'effectuer des mesures dendrométriques. Si c'était le cas, celles-ci devront être réalisées selon un protocole bien défini au préalable (ainsi une simple hauteur totale n'a aucun sens sans la connaissance de l'âge de l'arbre). Dans tous les cas, en raison de la très forte influence de la sylviculture passée sur l'état actuel d'un peuplement, ces mesures dendrométriques seront à manipuler avec précaution. Si la confrontation entre ces mesures et la typologie des stations est parfois intéressante, ces données ne doivent en aucun cas, peser sur l'élaboration de cette dernière.

Lors de la mise en place de la placette, il est nécessaire de s'assurer de l'homogénéité floristique (perception visuelle globale) et écologique (microtopographie en particulier) de celleci.

L'aire minimale du relevé floristique, en forêt, est le plus souvent de l'ordre de quatre ares; elle peut être légèrement augmentée pour les zones forestières à flore pauvre en espèces. Elle n'est pas obligatoirement circulaire, mais peut, par exemple, être déformée dans le sens des courbes de niveau.

L'étude des mosaïques, dans lesquelles le milieu et/ou la végétation présentent, de façon répétitive, des hétérogénéités importantes sur de très courtes distances, reste une question délicate. Pour la gestion, cette unité doit être considérée comme une station unique complexe ; mais au moment de l'étude de typologie, il y aura lieu de décrire séparément chaque élément de la mosaïque. Ceci est nécessaire pour analyser les corrélations entre les diverses caractéristiques de la station et les potentialités qui en découlent.

\section{Les méthodes d'analyse des données}

En raison de l'importance que peut prendre la masse des données recueillies pour une telle étude, la recherche des structures de la végétation et de leur déterminisme écologique ne peut se faire manuellement. On a de plus en plus recours aux outils mathématiques et informatiques.

Les techniques d'analyse statistique multivariable sont nombreuses, variées et plus ou moins adaptées à notre recherche. Il n'est pas dans notre propos de décrire des algorithmes de calcul et d'énumérer leurs avantages ou inconvénients.

Signalons simplement que, parmi celles-ci, il y en a trois qui sont largement utilisées en phytosociologie et en phyto-écologie et qui ont fait leurs preuves. II s'agit de l'analyse factorielle des correspondances, de la classification ascendante hiérarchique et de la méthode des profils écologiques.

\section{- L'analyse factorielle des correspondances (AFC)}

Assez généralement, l'analyse porte sur les espèces végétales par rapport aux relevés. Quelques essais ont été tentés en analysant les facteurs et descripteurs du milieu par rapport aux relevés ou ces facteurs par rapport aux espèces végétales; mais les résultats n'en sont pas encore très concluants.

Le plus souvent, l'analyse est effectuée avec les coefficients d'abondance-dominance des espèces. II apparait, en effet, qu'un tel traitement donne en général des résultats plus satisfaisants et plus précis, en particulier dans les milieux floristiquement pauvres.

On prendra garde aux espèces rares qui peuvent prendre un poids important dans le calcul des axes factoriels et entraîner des déformations. On veillera également à ne pas introduire de variables artificielles (espèces éclatées par coefficient d'abondance-dominance par exemple), qui perturberaient la logique de ce nuage. Ces variables sont à placer en variables supplémentaires. 


\section{A. BRÊTHES}

Les facteurs et descripteurs du milieu (caractères édaphiques, topographiques, etc...) seront eux aussi placés en variables supplémentaires. Ces variables ne participent pas au calcul des axes factoriels, mais sont replacées dans le nuage de points et projetées sur les divers plans factoriels. Elles facilitent ainsi l'interprétation écologique de ces axes et permettent de visualiser les corrélations entre groupements floristiques et les variables écologiques.

Dans la recherche de ces corrélations, les variables ayant un caractère intégrateur comme le type d'humus ou l'estimation de la réserve en eau, seront plus performantes que les variables élémentaires comme la classe de texture.

Plusieurs analyses successives, d'abord sur l'intégralité du fichier des données, puis sur des sous-ensembles de celui-ci, sont parfois nécessaires pour préciser les structures recherchées.

Rappelons enfin que l'analyse étant symétrique entre les variables analysées et les relevés, on obtient une structure comparable pour les premières et pour les seconds; ceci permet facilement leur mise en relation.

\section{- La classification ascendante hiérarchique (CAH)}

Celle-ci peut être effectuée directement sur le fichier de base, soit mieux encore, à partir des résultats de l'AFC (Buffet, 1984). Elle permet de mettre en évidence les rapprochements possibles entre espèces (établissement des groupes sociologiques) puis entre groupes d'espèces et, symétriquement, entre relevés puis entre groupes de relevés.

II est particulièrement intéressant de reporter ces résultats sur les plans factoriels obtenus cidessus et ainsi affiner la classification, en particulier à partir des différences qui peuvent apparaître.

\section{- Les profils écologiques}

S'ils ne peuvent servir de base à la recherche de la structure de la végétation, ils seront par contre, valablement utilisés pour apporter des précisions sur la valeur indicatrice des groupements établis.

\section{L'établissement de la typologie}

Ce travail est certainement le plus délicat. II demande une bonne connaissance de la région et de l'ensemble des facteurs qui jouent un rôle dans la différenciation des types de station.

Rappelons qu'il doit aboutir à deux résultats complémentaires :

- mettre en évidence les divers types de station existant dans la région et donner l'ensemble des caractéristiques de chacun d'eux;

- faire ressortir les facteurs simples permettant leur diagnostic et sur la base desquels seront ensuite construites la ou les clés d'identification.

La mise en forme de la typologie peut se résumer en trois étapes successives: analyse des relevés floristiques, définition des types de station en rapprochant les ensembles floristiques et les sols, recherche des éléments discriminants pour l'identification de la station.

- La structuration de la végétation

La structuration de la végétation a pour but essentiel de définir des ensembles d'espèces présentant des affinités de coexistence dans les mêmes stations.

Ces unités de végétation sont, dans la plupart des cas, interprétées selon l'une des deux démarches essentielles suivantes: 
- Elles sont rattachées à la systématique phytosociologique sigmatiste (associations, alliances et ordres). Pour pouvoir effectuer un diagnostic stationnel précis, il est, de plus, souvent nécessaire de définir des sous-associations et des variantes.

II conviendrait également de donner à ces unités phytosociologiques une interprétation écologique précise et de ne pas se limiter aux seuls termes classiques d'espèces différentielles, caractéristiques ou compagnes, peu évocateurs.

- On définit, pour la région étudiée, des "groupes écologiques" d'espèces dont les diverses combinaisons originales permettent de reconstituer la composition floristique moyenne des stations définies. Chaque groupe écologique traduit le rôle privilégié d'un facteur écologique déterminé.

II y a lieu de distinguer les groupes écologiques statistiques, au sens du Centre d'Études phytosociologiques et écologiques (CNRS) : un groupe rassemble les espèces présentant des amplitudes écologiques semblables à l'égard d'un ou plusieurs facteurs ou descripteurs écologiques préalablement choisis et mesurés systématiquement, et les groupes écosociologiques, au sens de Duvigneaud (1946) et Duchaufour (1960): on définit tout d'abord la sociologie des espèces puis on l'explique sur le plan écologique.

Dans ce qui suit, nous nous intéresserons uniquement à la définition et à l'utilisation de ces derniers groupes.

La constitution des groupes écologiques se basera sur les résultats des analyses précédemment citées (AFC et $\mathrm{CAH}$ ). Deux manières de procéder sont possibles:

- On recherche les coupures essentielles qui peuvent apparaître entre ensembles d'espèces sur les différents plans factoriels puis au sein de ces ensembles, des coupures secondaires, et ainsi de suite jusqu'au degré de précision souhaité. On évitera toutefois de définir des groupes à nombre d'espèces trop réduit (graphique 1, page 18).

Ces coupures sont d'autant plus faciles à fixer qu'il existe des discontinuités nettes dans la végétation; elles seront plus arbitraires si les groupements végétaux ont une large amplitude ou si les gradients écologiques sur le terrain sont très progressifs. Le tableau floristique dont nous parlerons plus loin est en général une aide à la recherche de ces limites.

- À partir d'espèces particulièrement signiticatives décelables dans l'AFC, on réalise des regroupements avec les espèces les plus proches, puis des regroupements entre groupes d'espèces. Ces agrégations successives doivent se faire sans perte d'information, c'est-à-dire, en veillant à ne pas regrouper des individus ou des groupes d'individus qui ne coexisteraient pas sur le terrain ou n'auraient pas la même écologie.

Les groupes ainsi obtenus sont d'abord sociologiques ; ils doivent être aussi écologiques, c'està-dire explicatifs. Il est en effet nécessaire de fournir à l'utilisateur des éléments permettant l'analyse du milieu et de ses caractères écologiques.

Il convient donc de déterminer les facteurs écologiques influençant la distribution de chaque espèce. Pour cela, on recherchera les corrélations entre ces espèces et groupes d'espèces et les divers facteurs et descripteurs du milieu (type d'humus, $\mathrm{pH}$, présence de calcaire, réserve en eau du sol, nature du matériau, exposition, etc...). L'utilisation de ces facteurs et descripteurs dans I'AFC est, de ce fait, quasi obligatoire. Elle sera valablement complétée par la réalisation de profils écologiques pour des espèces et des facteurs décelés comme les plus significatifs.

De manière assez générale, on peut considérer que quatre ensembles de facteurs régissent la répartition des diverses espèces : les facteurs édaphiques (richesse minérale et régime hydrique principalement), les facteurs climatiques, les facteurs biotiques liés à l'activité de certains animaux ou à l'action humaine, et les facteurs de dynamisme du milieu. On cherchera donc à 


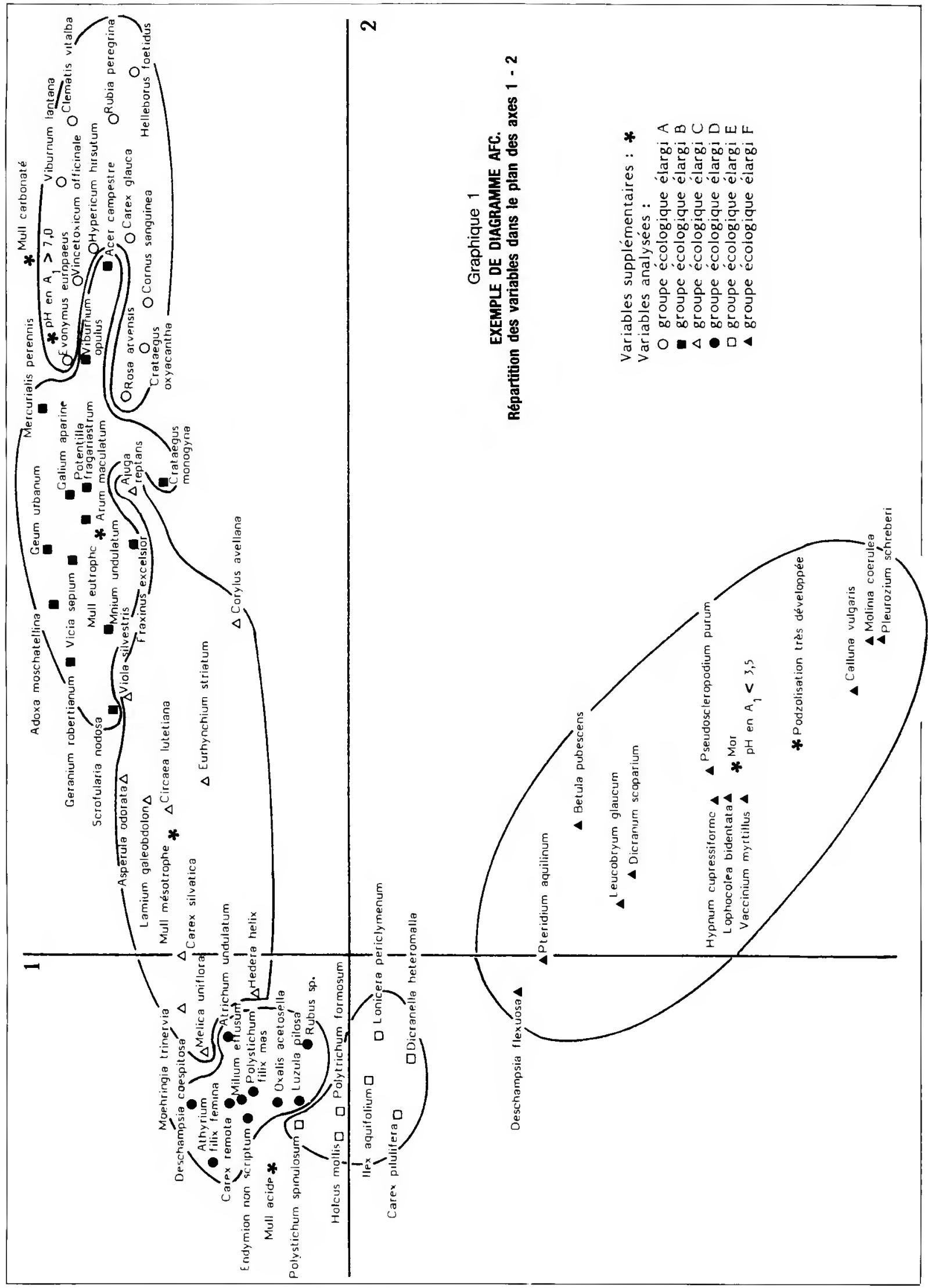




\section{La typologie des stations forestières. Recommandations méthodologiques}

mettre en évidence les divers groupes d'espèces permettant de faire un diagnostic au sein de ces grandes catégories, ainsi que les divers seuils au-delà desquels tel groupe disparaît ou apparaît : seuil de fertilité ou de toxicité (aluminium en milieu acide, calcaire), de réserve en eau ou d'engorgement, etc...

\section{- Définition des types de station}

Après avoir établi les groupes sociologiques et définit leurs caractéristiques écologiques, il faut de la même manière définir les ensembles de relevés caractérisés par un groupement floristique analogue et des caractères écologiques comparables.

Les groupes de relevès

De la même manière qu'ont été formés des groupes d'espèces, il est possible, à partir des résultats de l'AFC et de la $\mathrm{CAH}$, de définir des ensembles de relevés.

En raison de la symétrie des résultats de ces analyses, ces groupes de relevés peuvent être mis en corrélation directe avec les groupes d'espèces définis ci-dessus. On met ainsi en évidence les relevés qui sont à l'origine des divers axes factoriels et les ensembles ayant une composition floristique similaire.

Le tableau floristique

La réalisation d'un tableau ordonné à double entrée (relevés $\times$ espèces), permet de mettre en évidence les ensembles floristiques homogènes (graphique 2, page 20-21).

L'élaboration de ce tableau est facilitée par les analyses statistiques précédentes: les espèces sont classées en groupes écologiques selon le gradient apparu comme le plus important (en général l'axe 1 de l'AFC); les ensembles de relevés sont, en première approximation, placés dans l'ordre où ils apparaissent sur ce même gradient.

Par retouches successives, en déplaçant les lignes et les colonnes, on cherche à "diagonaliser " ce tableau, c'est-à-dire que l'on se propose tout d'abord de regrouper les relevés possédant la même combinaison de groupes écologiques, puis d'ordonner ces ensembles selon la diagonale du tableau. On visualise alors des marches d'escalier successives en tonction de l'apparition ou de la disparition de groupes écologiques.

La diagonale du tableau ne pouvant représenter qu'un gradient, il est nécessaire de créer des discontinuitès dans cet ordonnancement pour taire apparaître les autres facteurs importants. Ainsi, le premier gradient peut correspondre à la richesse minérale (alimentation minèrale, milieux calcaires - milieux acides, type d'humus ...) - c'est le cas le plus fréquent en plaine et les discontinuités à la réserve en eau des sols (milieux secs, frais et engorgés par exemple).

Les types de station

Les analyses statistiques et le tableau floristique permettent la constitution d'ensembles de relevés floristiquement homogènes. Ce ne sont pas pour autant encore des types de station.

Rappelons qu'un type de station doit regrouper des stations (représentées par les relevés) possédant à la fois une combinaison originale de groupes écologiques, un sol similaire et situés dans un même environnement géomorphologique.

Au sein de ces ensembles de relevés, on est en général amené à faire des subdivisions pour obtenir des sous-ensembles homogènes également quant à leurs caractéristiques écologiques (Brêthes, 1988). 


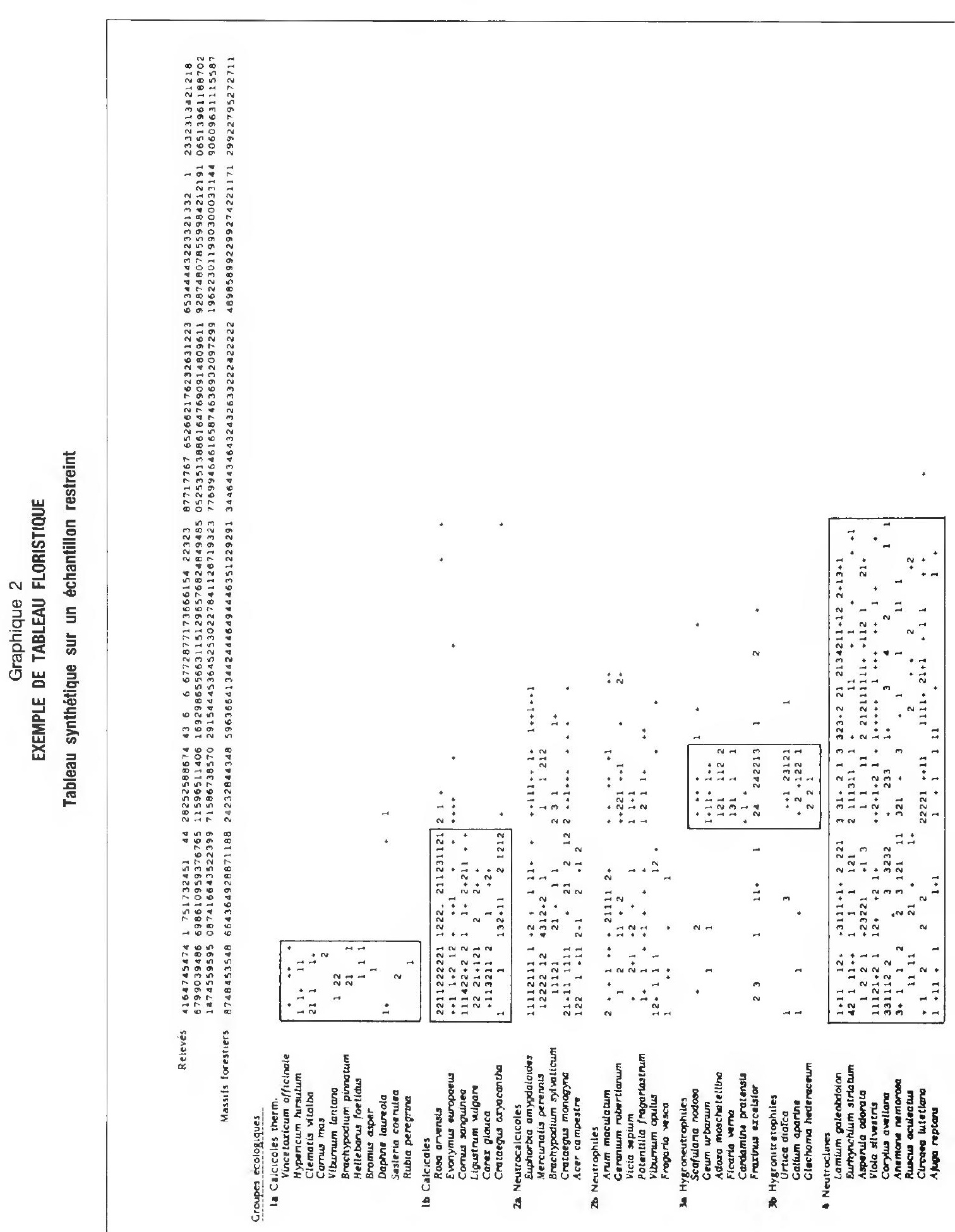




\section{A. BRÊTHES}

Mais aucune station n'est parfaitement identique à une autre; le fait de regrouper certaines d'entre elles au sein d'un même type nécessite de faire abstraction d'un certain niveau d'hétérogénéité que l'on jugera acceptable. II y a lieu pour cela de respecter certaines règles :

- Homogénéitè de la position topographique et géomorphologique, car celle-ci conditionne, entre autres, le climat, le drainage naturel, la réserve en eau.

- Homogénéité du sol: les différences pouvant exister entre les profils pédologiques des stations d'un même type ne doivent pas avoir un effet sur la potentialité de la station. On retiendra parmi les critères édaphiques de potentialité : la profondeur facilement prospectable par les racines, la réserve en eau (fonction de cette profondeur, de la texture, de la charge en cailloux, de la structure et de la compacité du sol), la fertilité (reflétée en particulier par le type d'humus), les éléments de contraintes comme l'hydromorphie, la présence de calcaire, l'existence d'un niveau induré, etc... (Duchaufour, 1986).

- Homogénéité floristique: cette homogénéité ne peut se concevoir qu'à structure et composition du peuplement similaires. On connaît l'appauvrissement de la végétation en milieu très fermé, la banalisation du tapis végétal dans les peuplements monospécifiques et même la modification de ce cortège végétal dans les peuplements artificiels de résineux. La typologie doit d'ailleurs faire apparaître tous les sylvofaciès d'un même type de station.

Dans tous les cas, il est nécessaire que soit précisée, dans la fiche d'identité du type de station, la variabilitè, tout autant de la flore, que du sol qui aura été reconnue et acceptée. Ceci laisse ainsi la possibilité d'effectuer ultérieurement de nouvelles divisions qui s'avéreraient nécessaires.

Il est souhaité que cette définition des types de station soit faite avec le maximum de précision possible. On décrira donc les unités élémentaires existantes sans préoccupation de leur utilisation sylvicole future. Ainsi, il est hors de question de regrouper deux types de station quelque peu différents parce qu'ils semblent convenir tous deux aussi bien au Chêne qu'au Douglas, même si cela était confirmé, le même regroupement peut être contestable pour une autre essence.

Par contre, il importe de présenter les apparentements décelés entre types de station car l'utilisation cartographique de la typologie dans le cadre de la gestion forestière nécessite de définir des unités de surface suffisante; ces unités pourront résulter alors du regroupement raisonné de certains types de station en fonction d'un objectif précis. Ultérieurement, ces regroupements pourront être différents si les objectifs changent; mais la typologie initiale, elle, demeurera stable.

\section{Les sylvofaciès}

La réalisation de la typologie telle que nous venons de l'évoquer serait relativement aisée si un type de station donné était toujours caractérisé par le même cortège végétal (même si ce cortège est partagé par plusieurs types de station). Si ceci est souvent vrai pour les forêts relativement proches de leur climax, ce n'est plus le cas pour leurs sylvofaciès.

La caractérisation de ces derniers est plus délicate. On partira du principe que, pour tous les sylvofaciès d'un même type de station, les caractéristiques essentielles du sol sont peu modifiées et qu'en tout état de cause ces modifications n'influent pas sur la potentialité forestière et sont réversibles (condition sine qua non pour rattacher ces sylvofaciès à un même type de station).

L'identification de groupes d'espèces exprimant l'évolution du peuplement ou de groupes écologiques analogues (groupes d'espèces diffèrents dans leur composition mais reflétant les mêmes propriétés du milieu) peut aider valablement cette recherche. 


\section{- Les éléments de caractérisation des types de station}

Il importe de rappeler la distinction entre les véritables facteurs discriminants qui font qu'un type de station est différent d'un autre et les éléments de caractérisation (ou descripteurs) qui sont l'expression de ces différences. L'analyse des données doit permettre de comprendre les premiers et de mettre en évidence les seconds.

Les descripteurs retenus devront être aussi simples que possible et facilement perceptibles afin que l'identification du type de station soit réalisable par tous après un minimum de formation et que l'application cartographique soit rapide.

On songera tout d'abord à l'utilisation de la végétation, la présence de certaines espèces ou de certains groupes écologiques pouvant être plus ou moins discriminante. Ceci facilitera tout particulièrement la réalisation des travaux cartographiques.

Mais la végétation seule est en général insuffisante et il convient de se tourner aussi vers les descripteurs physiques du milieu, et en particulier vers les critères édaphiques. La tarière pédologique est alors bien souvent nécessaire.

On ne peut dresser, a priori, la liste des caractères diagnostiques utiles et encore moins donner leur hiérarchie ; ils dépendent essentiellement du contexte régional.

\section{La présentation des résultats}

Nous ne reviendrons pas sur l'ensemble du contenu que doit comporter un catalogue des stations forestières. On se référera pour cela à la brochure de J. Timbal (1984).

Il y a lieu toutefois de préciser trois points importants de ce document :

\section{- La fiche d'identité du type de station}

Cette fiche d'identité doit contenir tous les éléments nécessaires d'une part au diagnostic du type de station, d'autre part à l'appréciation de sa valeur forestière.

Pour ce second aspect, on songera tout particulièrement à souligner certaines propriétés fondamentales du type de station (réserve minérale et hydrique, éléments de contraintes particuliers) et les améliorations possibles ; il convient de préciser également la sensibilité de la station à l'érosion, aux aléas climatiques ou aux diverses actions humaines en particulier aux pratiques sylvicoles.

Les caractères de la station évoluant peu dans le temps, du moins à l'échelle humaine, un tel document aura une validité quasi permanente. On prendra garde à ne pas donner à ce niveau des informations qui seraient rapidement caduques. Ainsi toutes indications dendrométriques (hauteurs, production) ne seront indiquées que dans la mesure où une étude détaillée aura été réalisée. Mais comme ces données elles-mêmes peuvent être précisées et complétées par la suite, il semble préférable d'y consacrer un chapitre distinct de la description proprement dite des types de station.

Toutefois la fiche d'identité doit contenir des renseignements sur les peuplements effectivement rencontrés sur ces stations (composition, structure, état sanitaire, etc...), en particulier lorsqu'il existe des sylvotaciès variés.

\section{- La clé d'identification des types de station}

Un catalogue des stations, aussi précis soit-il, ne pourra être valablement utilisé que s'il comporte une ou plusieurs clés de détermination des types de station sur le terrain. 


\section{A. BRÊTHES}

Ces clés devront être simples, fiables et opérationnelles. Elles seront construites à partir des meilleurs caractères diagnostiques mis en évidence. On y évitera les caractéristiques trop floues, obligeant l'utilisateur à une trop grande part d'interprétation personnelle, qui peuvent devenir source d'erreur.

S'il peut exister une certaine concordance entre la hiérarchie des divers types de station et celle des éléments descriptifs dans la clè, cette concordance n'est pas obligatoire. II peut être souvent plus judicieux de commencer par des facteurs ou descripteurs facilement et immédiatement observables (topographie, exposition...) pour ensuite affiner la détermination avec les éléments floristiques et/ou édaphiques.

Les utilisateurs potentiels de cette clé peuvent avoir une prédilection plus grande pour la vègétation ou pour la pédologie. On songera donc à réaliser non pas une mais deux clès: l'une à dominante floristique, l'autre à dominante pédologique, l'une et l'autre restant toutefois le plus souvent mixtes, sol et végétation intervenant à des niveaux différents.

Soulignons que les informations pédologiques sont indispensables pour caractériser des types de station dans des peuplements très fermès ou dans certains sylvofaciès dont la végétation est fortement appauvrie ou modifiée.

- Le classement hiérarchisé des types de station

Comme nous l'avons indiqué plus haut, le corollaire de la précision maximale demandèe dans la typologie est un classement hiérarchisé des types de station. En effet, les impératifs d'échelle cartographique, de surface de gestion, etc... nécessitent souvent d'effectuer des regroupements entre certains d'entre eux.

Trois modes de classement peuvent être retenus pour ces regroupements :

- Un classement naturel qui correspond aux potentialités climaciques. II repose sur l'ensemble des facteurs écologiques (topographie, similitude des matériaux parentaux, type d'humus, groupement végétal, etc...); il est de ce fait unique.

Ce classement, qui permet des regroupements par similitude de potentialités, doit figurer dans le catalogue des stations.

- Un classement par proximité gèographique : dans la mesure où certains types de station, même différents, se retrouvent toujours dans la même disposition spatiale, par exemple le long de toposéquences ou en mosaïques, on peut songer à faire des unités complexes plus faciles à faire figurer sur une carte.

Ce mode de regroupement peut également figurer dans le catalogue. Mais il faut souligner qu'il ne s'agit là que de faciliter le tracé d'une carte ; pour la gestion, il est nécessaire de revenir à la station élémentaire.

- Un classement strictement conçu pour la gestion, qui regroupe tous les types de station ayant des potentialités identiques pour une essence donnée.

Ce dernier type de classement est éminemment variable; toutes les possibilités ne peuvent donc figurer dans le catalogue, lequel retiendra seulement quelques suggestions pour ètablir ces regroupements. Ceux-ci doivent être construits en comparant l'autécologie de l'essence concernèe aux caractéristiques des types de station.

\section{Tests et vulgarisation}

Avant la rédaction définitive d'un catalogue, il est indispensable de mettre à l'épreuve la typologie proposèe et de tester la pertinence de la (ou des) clé(s).

Pour cela on pourra procéder de deux manières : 
- par des diagnostics ponctuels en des points choisis au hasard et, si possible, par une personne n'ayant pas ou peu participé à l'élaboration de la typologie ;

- par une cartographie de portions de forêts non visitées lors de l'échantillonnage et susceptibles de présenter une assez grande diversité de types de station.

Ces tests peuvent amener à modifier les clés proposées pour les rendre plus opérationnelles.

Mais un catalogue ne passera dans la pratique que si ses utilisateurs potentiels sont initiés à son utilisation. Pour cela, le réalisateur du catalogue, en liaison avec les divers services forestiers concernés, devra obligatoirement assurer une information lors de l'élaboration de l'étude puis la mise en place de sessions pratiques de formation.

Divers schémas peuvent d'ailleurs être imaginés, depuis la simple journée d'information jusqu'au stage pratique de cartographie.

\section{CONCLUSIONS}

Grâce à l'ensemble des études déjà réalisées et au progrès des discussions entre scientifiques et forestiers, on peut considérer que la typologie des stations a atteint une certaine maturité et qu'elle est sortie du domaine de la recherche pour devenir une technique opérationnelle.

Tout au long de ces rẻalisations et discussions, les méthodes se sont précisées, affinées et même simplifiées. La typologie des stations forestières est, en effet, une recherche finalisée, c'est-à-dire que l'utilisateur doit trouver dans ses résultats les éléments de diagnostics stationnels dont il a besoin, au travers d'un document simple et clair, mais complet.

II est important de souligner que la qualité et la précision du document obtenu dépendent en grande partie de celles des données recueillies sur le terrain. Le fait de préciser les éléments obligatoires à prendre en compte ne signifie pas que la méthode soit figée et que toutes les régions doivent être étudiées dans le détail de la même manière.

La définition d'un type de station repose toujours sur la connaissance des trois éléments: mésoclimat, végétation, sol. L'originalité d'une région donnée s'exprime dans celle des relations entre ces trois éléments et dans la nature des caractères diagnostiques les plus discriminants.

La présentation du catalogue des stations forestières appelle deux remarques:

- Dans la mesure où l'on y met les moyens en hommes, en temps et en argent, la définition des types de station doit être aussi précise que possible, même si tous les résultats ne sont pas valorisés dans l'immédiat. Le catalogue constitue une référence permanente pour tout ce qui touche à l'aménagement du milieu forestier.

- Les modèles de sylviculture se modifient régulièrement, l'autécologie des essences se précise de jour en jour, de même que les méthodes d'amélioration des sols. Au fil de l'évolution normale de ces progrès, on devrait trouver dans les catalogues de stations les informations encore inexploitées qui permettront de valoriser au mieux ces connaissances nouvelles.

On peut d'ailleurs songer à publier outre le catalogue des stations, ouvrage à bases essentiellement scientifiques, et au fur-et-à-mesure du développement des connaissances, des fascicules sur le choix des essences selon la nature des stations, sur les productions par essence et par station, sur les conseils de sylviculture, etc.

Quel que soit le moment où sont réalisées ces études de peuplements, il importe que ce soit selon un protocole précis mis au point avec des dendrométriciens. Leur réalisation conjointe 


\section{A. BRÊTHES}

avec celle des stations n'est d'ailleurs pas conseillée (alourdissement de la phase de terrain, critères de choix des placettes différents, périodes favorables d'observation décalées, etc...)

Grâce à l'expérience acquise depuis quelques années, il apparaît désormais possible de proposer une méthode d'étude des stations fiable, rapide à mettre en place et à réaliser. Elle nécessite toutefois une expérience certaine de la part du chargé d'études.

Les techniques éprouvées jusqu'ici ont surtout fait leurs preuves dans les forêts déjà bien constituées et dont les peuplements sont dans un équilibre relatif avec les conditions du milieu. Pour les formations dejgradées et/ou plus ou moins ouvertes (landes, friches, prairies...), la méthodologie mérite d'être encore largement approfondie.

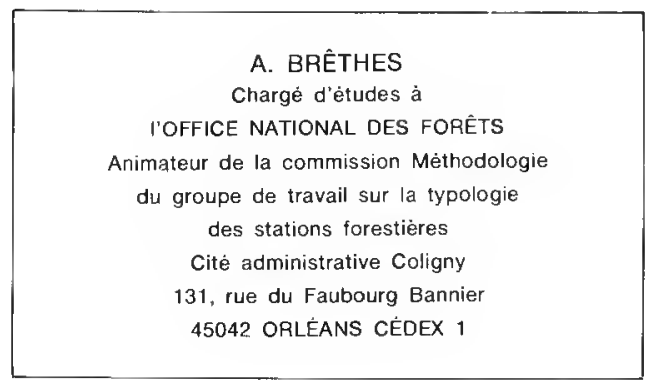

\section{Remerciements}

Nous tenons à remercier Messieurs Michel Becker de l'Institut national de la Recherche agronomique (INRA) et Gérard Dumè de l'Institut pour le Développement forestier (IDF) pour leur collaboration à la mise au point de cet article.

\section{BIBLIOGRAPHIE}

BECKER (M.). - Avantages et limites de l'ètude de la vėgétation spontanée pour la typologie des stations forestières. - Comptes rendus des Séances de l'Acadẻmie d'Agriculture de France, vol. $72, \mathrm{n}^{\circ} 10,1986$, pp. $875-882$.

BECKER (M.). - Démarche mëthodologique préconisèe pour la typologie des stations forestières. In : Colloques phytosociologiques, XIV, "Phytosociologie et foresterie ", Nancy, novembre 1985. - 1988, pp. 299311.

BECKER (M.), LE GOFF (N.). - Diagnostic stationnel et potentiel de production. - Revue forestière française, numéro spécial "Diagnostics en forêt", vol. XL, 1988, pp. 29-43.

BRÊTHES (A.). - La Typologie des stations forestières en Haute-Normandie; apports complèmentaires du sol et de la végétation. In: Colloques phytosociologiques, XIV, "Phytosociologie et foresterie", Nancy, novembre 1985. - 1988, pp. 435-466.

BUFFET (M.). - La Description du milieu pour l'amènagement des forêts ; application d'un algorithme de classification à la recherche d'une typologie de stations. - pp. 31-38. In : Symposium Aménagement et Gestion (IUFRO), Nancy, 7-11 mai 1984. - Nancy: Ėcole nationale du Génie rural, des Eaux et des Forêts, 1984. - $127 \mathrm{p}$.

DELPECH (R.), DUMÉ (G.), GALMICHE (P.). - Typologie des stations forestières, vocabulaire. - Paris : Ministère de l'Agriculture, Institut pour le Dèveloppement forestier, 1985. - $243 \mathrm{p}$. 
La typologie des stations forestières. Recommandations méthodologiques

DUCHAUFOUR (Ph.). - Stations, types d'humus et groupements écologiques. - Revue forestière française, vol. XII, $n^{\circ} 7,1960$, pp. 484-494.

DUCHAUFOUR (Ph.). - La Typologie des stations forestières: utilisation des données de la pèdologie. Comptes rendus des Séances de l'Académie d'Agriculture de France, vol. 72, $\mathrm{n}^{\circ} 10,1986, \mathrm{pp} .883-891$.

DUMĖ (G.). - Activitès et résultats du groupe de travail sur la typologie des stations forestières. In : Colloques phytosociologiques, XIV: "Phytosociologie et foresterie ", Nancy, novembre 1985. - 1988, pp. $289-298$.

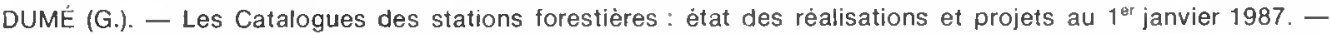
Forêt-Entreprise, $\mathrm{n}^{\circ} 41,1987$, pp. 6-11.

DUMÉ (G.). - Guide-âne pour la rèalisation d'un catalogue des stations forestières. - Forêt-Entreprise, $\mathrm{n}^{\circ} 19$, 1984, pp. 34-38.

DUVIGNEAUD (P.). - La Variabilité des associations vègètales. - Bulletin de la Sociètè royale de Botanique de Belgique, tome 78, 1946, pp. 107-134.

GROUPE DE TRAVAIL sur la typologie des stations forestières. Commission Méthodologie. - Comptes rendus des diverses rèunions tenues de 1980 à 1987.

RAMEAU (J.-C.). - Les Ėtudes stationnelles forestières en France. - Nancy : École nationale du Génie rural, des Eaux et des Forêts - Laboratoire de Botanique et Phytosociologie forestière, 1986. - $90 \mathrm{p}$.

TIMBAL (J.). - Recommandations pour la présentation des catalogues de stations forestières. - Paris Ministère de l'Agriculture - Direction des Forêts, 1984. - 41 p.

\section{LA TYPOLOGIE DES STATIONS FORESTIĖRES. RECOMMANDATIONS MÉTHODOLOGIQUES (Résumé)}

La rèalisation d'une étude régionale de typologie des stations forestières, si l'on veut qu'elle réponde bien à l'attente des gestionnaires-sylviculteurs, doit respecter un certain nombre de principes, essentiellement quant à l'échantillonnage et à la présentation des résultats.

Notre propos n'est pas d'imposer une mèthode applicable par tous et partout, mais de rappeler les èlements indispensables pour que cette typologie permette à la fois une caracterrisation précise des divers types de station et la compréhension des propriètés fondamentales de ces stations vis-à-vis de la mise en valeur forestière.

Dans cet article, sont d'abord rappelées, succinctement, les notions de région naturelle, de station forestière, de type de station at de sylvofaciès. Puis sont abordès les points fondamentaux de la démarche typologique : preétude régionale, mode et contenu de l'èchantillonnage phytoécologique, principes de dépouillement des donnèes et d'établissement de la typologie des statıons, contenu du catalogue des stations forestieres.

\section{TYPOLOGY OF FOREST SITES. RECOMMENDATIONS ON METHODOLOGY (Summary)}

The execution of a regional study of the typology of forest sites, if it is to fulfill the expectations of the forest managers, should follow a certain number of principles, mainly in respect of sampling and the presentation of results.

Our aim is not to set out a method which can be applied by all to all cases, but to review the essential elements so that the typology makes it possible both to precisely define the various site types and to understand the lundamental properties of these sites in relation to forest utilisation.

In this article a briet review is tirst given of the concepts of natural region, forest site, site type and sylvotacies. This is followed by a study of the fundamental points in the typological process: praliminary regional assessment, method and content of the phytoecological sampling. principles of the analysis of the data and of the establishment of the typology of the sites, contents of the list of forest sites.

\section{DIE TYPOLOGIE DER FORSTLICHEN STANDORTE. METHODOLDGISCHE EMPFEHLUNGEN (ZusammEnfasSUng)}

Die Durchführung einer regionalen Untersuchung der verschieden Typen der forstlichen Standorte muß eine gewisse Anzahl von Prinzipien besonders im Hinblick auf die Probennahme und die Darstellung der Ergebnisse respektieren, wenn sie den Erwartungen der Forstleute Rechnung tragen will.

Es ist nicht unsere Absicht, eine für alle und überall anwendbare Methode durchzusetzen, sondern die unbedingt notwendigen Elemente aufzuzählen, damit diese. Typologie es erlaubt, zugleich die verschiedenen Standorttypen zu charakterisieren und die fundamentalen Eigenschaften dieser Standorte hinsichtlich der forstlichen Erschließung zu begreifen.

In diesem Artikel wird zunächst kurz auf die Begriffe natürliche Gegend, Standort, Standorttyp und Forstfazies eingegangen. Danach werden die Hauptpunkte des typologischen Vorgehens erörtet: regionale Voruntersuchung, Art und Inhalt der phytoökologischen Probennahme, Prinzipien, der Datenauswertung und der Erstellung der Standorttypologie, Inhalt des Verzeichnisses der forstlichen Standorte.

\section{LA TIPOLOGIA DE LAS ESTACIONES FORESTALES. RECOMENDACIONES METODOLOGICAS (RESUMEN)}

La realizaciòn de un estudio regional de tipologia de las estaciones forestales, si se quiere que responda bien a la demanda de los cuestionarios-silvicultores, debe respetar cierto número de principios, esencialmente en cuanto al muestrario y a la presentación de los resultados.

Nuestro propósito no es imponer un mètodo aplicable por todos y por todo, sino recordar los elementos indispensables para que esa tipologia permita a la vez una caracterización precisa de los diversos tipos de estación y la comprensión de las propiedades fundamentales de dichas estaciones, con respecto a la puesta en valor forestal.

En este articulo son evocadas, sucintamente, las nociones de región natural, de estación forestal, de tipo de silvofacies. Después son abordados los puntos fundamentales del procedimiento tipológico : estudio previo regional, modo y contenido del muestrario fitoecológico, principios del examen de los datos y establecimiento de la tipologia de las estaciones, contenido del catálogo de las estaciones lorestales. 